Supporting Information - Revised ES0610479.

\title{
Leaching Assessments of Hazardous Materials in Cellular Telephones
}

\author{
JOHN D. LINCOLN ${ }^{1}$, OLADELE A. OGUNSEITAN ${ }^{* 2}$, ANDREW A. SHAPIRO ${ }^{3}$ and JEAN-DANIEL M. \\ SAPHORES $^{4}$
}

This supporting information includes two Tables. Table A, on two pages, presents the salient characteristics of cellular phones used in the study. Table B presents the salient parameters of leaching tests. 
Supporting Information - Revised ES0610479.

Table A. Salient Characteristics of Cellular Phones Used in the Study*

\begin{tabular}{|c|c|c|c|}
\hline Manufacturer & Model & Weight (g) & Volume $\left(\mathrm{cm}^{3}\right)$ \\
\hline Motorola & Unspecified & 86.56 & 94.75 \\
\hline Motorola & Unspecified & 87.30 & 94.75 \\
\hline Motorola & T720c & 87.02 & 88.42 \\
\hline Motorola & T720c & 88.90 & 88.42 \\
\hline Motorola & 80148WNBEA & 84.24 & 94.75 \\
\hline Motorola & Unspecified & 88.19 & 94.75 \\
\hline Motorola & Unspecified & 94.99 & 175.97 \\
\hline Motorola & $120 \mathrm{E}$ & 75.21 & 87.73 \\
\hline Sony/Ericsson & R278d & 84.83 & 151.50 \\
\hline Sony/Ericsson & T61c & 107.38 & 105.20 \\
\hline Sony/Ericsson & T61d & 91.24 & 119.02 \\
\hline Sony/Ericsson & T60d & 93.33 & 119.02 \\
\hline Sony/Ericsson & CM-B1201SPR & 113.90 & 117.37 \\
\hline Sony/Ericsson & R300d & 84.32 & 105.52 \\
\hline Sony/Ericsson & A1228di & 86.01 & 154.03 \\
\hline Sony/Ericsson & R278d & 85.37 & 151.62 \\
\hline Sony/Ericsson & R289LX & 84.79 & 101.82 \\
\hline Nokia & $282 \mathrm{~N}$ & 85.15 & 106.88 \\
\hline Nokia & 3585 & 79.53 & 79.27 \\
\hline
\end{tabular}


Supporting Information - Revised ES0610479.

\begin{tabular}{|c|c|c|c|}
\hline Nokia & 282 & 84.05 & 114.80 \\
\hline Mitsubishi & G310 & 82.84 & 97.99 \\
\hline Mitsubishi & $\mathrm{T} 200$ & 94.19 & 110.80 \\
\hline Mitsubishi & T300 & 87.06 & 162.10 \\
\hline Mitsubishi & G100E & 105.59 & 148.23 \\
\hline Samsung & $\mathrm{SCH}-211$ & 101.25 & 116.02 \\
\hline Samsung & SCH-2000 & 95.22 & 148.59 \\
\hline Sprint PCS & TP2200 & 89.85 & 93.65 \\
\hline Sprint PCS & TP2200 & 89.57 & 90.90 \\
\hline Qualcomm & QCP820 & 109.43 & 143.64 \\
\hline Audiovox & CDM 4000XL & 91.95 & 150.84 \\
\hline Audiovox & CDM 4000A & 93.31 & 125.52 \\
\hline Sharp & Z-800 & 95.27 & 131.55 \\
\hline Toshiba & CDMA 2000 & 98.57 & 122.71 \\
\hline Uniden & MINI 1200 & 90.52 & 91.22 \\
\hline
\end{tabular}

*A total of 148 phones were evaluated with average weight of $92.82 \mathrm{~g}$ (Standard Deviation $=$ $22.20 \mathrm{~g}$ ), and volume of $123.2 \mathrm{~cm}^{3}\left(\right.$ Standard deviation $\left.=39.02 \mathrm{~cm}^{3}\right)$. The 34 phones selected for the study had dimensions within one standard deviation of the mean for all dimension. 
Supporting Information - Revised ES0610479.

Table B. Salient Parameters of Leaching Tests.

\begin{tabular}{|c|c|c|c|c|c|c|}
\hline $\begin{array}{c}\text { Test } \\
\text { Procedure }\end{array}$ & $\begin{array}{l}\text { Regulatory } \\
\text { Jurisdiction }\end{array}$ & $\begin{array}{l}\text { Leaching } \\
\text { Solution }\end{array}$ & pH & $\begin{array}{c}\text { Particle } \\
\text { Size } \\
\text { (mm) }\end{array}$ & $\begin{array}{c}\text { Dilution } \\
\text { Factor }\end{array}$ & $\begin{array}{c}\text { Extraction } \\
\text { Time (h) }\end{array}$ \\
\hline TCLP & $\begin{array}{c}\text { Federal } \\
\text { (USA) }\end{array}$ & $\begin{array}{l}\text { Acetic } \\
\text { Acid }\end{array}$ & 4.9 & $<9.5$ & $20: 1$ & 18 \\
\hline TTLC & California & Nitric Acid & $<1.0$ & $\begin{array}{c}<2.0 \\
\text { (Inorganic } \\
\text { compounds) } \\
<1.0 \\
\text { (Organic } \\
\text { compounds }\end{array}$ & $15: 1$ & 0.5 (Reflux) \\
\hline WET & California & $\begin{array}{c}0.2 \mathrm{M} \\
\text { Sodium } \\
\text { Citrate }\end{array}$ & 5.0 & $<2.0$ & 10:1 & 48 \\
\hline
\end{tabular}

